\title{
Dental Implant Monitoring Using Resonance Frequency Analysis (RFA) and Cone Beam Computed Tomography (CBCT) Measurement
}

\author{
Maya Genisa ${ }^{*}$, Solehuddin Shuib ${ }^{2 *}$, Zainul Ahmad Rajion ${ }^{3}$, Dasmawati Mohamad ${ }^{4}$, \\ Erry Mochamad Arief ${ }^{4}$ \\ 1 Magister Sains Biomedis, Universitas YARSI, Jl. Letjend Suprapto No.13, Cempaka. Putih, Jakarta 10510, \\ Indonesia \\ ${ }^{2}$ Faculty of Mechanical Engineering, Universiti Teknologi MARA, Jalan Ilmu 1/1, Shah Alam, Selangor 40450, \\ Malaysia \\ ${ }^{3}$ Kulliyyah of Dentistry, International Islamic University Malaysia, Jalan Sultan Ahmad Shah, Bandar Indera \\ Mahkota, Kuantan 25200, Malaysia \\ ${ }^{4}$ School of Dental Sciences, Universiti Sains Malaysia, Jalan Raja Perempuan Zainab 2, Kota Bharu, Kelantan \\ 16150, Malaysia
}

\begin{abstract}
Dental implants commonly practiced replacing edentulous teeth. However, it is still challenging to evaluate the progress of osseointegration during the healing process after implant placement. This study aims to measure the implant stability of pre- and post-crown placement to monitor osseointegration during the healing process and correlate it with the bone quality and quantity and other parameters. Resonance Frequency Analysis (RFA) method as a standard method has been used to monitor implant stability. Ten patients from Hospital Universiti Sains Malaysia have been involved with and treated by immediate implant placement procedures on their mandibular jaw system. Monitoring was also conducted by measuring the density of bone estimated based on Cone Beam Computed Tomography data. On the basis of the study, RFA and density monitoring show that there are at least three classes of patients: Class 1 are the patients who have a significant increment of implant stability; Class 2 are the patients with constant implant stability; and Class 3 are the patients who have negative implant stability progress, or their implant stability was decreasing. On the basis of the result, monitoring of implant stability by measuring the density is still challenging, the correlation between secondary implant stability and density is not significant statistically. It is recommended in future research to evaluate the implant stability by involving more patients from different races and also correlating the implant stability with the dynamic properties such as stress distribution.
\end{abstract}

Keywords: CBCT; Density; Dental implant; Osseointegration; RFA

\section{Introduction}

In the last decade, dental implant treatment has become more popular and it practiced protecting the remaining teeth, improving mastication, and also the appearance of the patient. The primary purposes of dental implant placement are to replace the edentulous tooth (Supriadi et al., 2015). However, a dental implant was planted not only to improve

*Corresponding author's email: maya.genisa@yarsi.ac.id, solehuddin2445@uitm.edu.my Tel.: +62-21-4206675, Fax: +62-21-4243171

doi: 10.14716/ijtech.v11i5.4326 
appearance, but also to improve the masticatory function and to prevent the changes of the dental arch dimension or to restore facial skeletal structure (Turkyilmaz and Mcglumphy, 2008).

Staden et al. (2006) showed that the success rate of dental implants is over 95\%, and the survival rate at 15 years is about 90\% (2006). However, the compatibility of the dental implant might generate some problems for a specific patient, such as in conveying the force during mastication. As the dental implant is not a natural tooth, some patients need adaptation until the jawbone system accepts the implanted biomaterial to experience more convenience. The integration of the implanted biomaterial into the jawbone system is called as osseointegration.

During the healing process, where osseointegration is still not achieved, some problems might appear to particular patients, such as infection or mechanical problem due to an inadequate load protocol. For example, in the case where the implant is not placed correctly, or the shape of the implant is not suitable, it would not appropriately couple into the jawbone. In such a case, a space will be generated between the surface of the implant and the bone, which can be potentially used by bacteria to grow. The problem might become more severe if the daily mastication also promotes the immobility of the implant and holes. Daily mastication will also generate stress in the implant body and bone. If the generated stress is too low, the stimulus of osteoblast activity required for bone growth might also be delayed. Again, if the generated stress is too high, then the stability of the implant might be disturbed, thus affecting the osseointegration process (Chang et al., 2010). Hence, the monitoring of osseointegration becomes the most critical task, especially during the healing period, to ensure the success of implant treatment.

Implant stability as an indicator of the immobility of an implant is measured directly through clinical measurement (Rabel et al., 2007). On the basis of the occurrence, implant stability is categorized into primary implant stability, which is the stability achieved during implant placement, and secondary implant stability, which is reached after implant placement. Many factors affect the implant stability, including the material of implant used, size, length, and diameter of the implant, and internal factors such as bone conditions and health. The most critical internal factor determining implant stability is the quality and quantity of the bone and osseointegration process (Rabel et al., 2007).

Implant stabilities have been evaluated using different methods such as pull in, push out, cutting torque resistance, reverse torque test, and percussion test. However, all these methods are categorized as destructive methods which are only available for preclinical usage (Swami et al., 2016). In clinical usage, non-destructive methods such as radiographic (Computed Tomography (CT), CBCT) and RFA methods are used.

This article discusses the periodic monitoring of implant stability of mandibular cases during the healing process using a non-destructive method (i.e., RFA technique) on the three different stages (immediate, three months, and four months after implant placement). Measurements were conducted once at pre-crown conditions, and the other two stages are conducted at post-crown conditions. A comparative study between the different stages is undertaken to evaluate the progress of the osseo-integration process. Statistical analysis is also conducted to assess the relationship between implant stability and other bone quality and quantity. The current measurement of the implant is monitored by a clinician in a regular check-up without considering the detail stage of healing process. In this research, implant stability monitoring is conducted by considering the condition of the implant in relation to the stages (four stages). 


\section{Methods}

The clinical treatments involving dental implant patients and a series of laboratory works have been conducted. Ten patients were selected to be involved in this research, with the ethical approval being obtained from Hospital Universiti Sains Malaysia (Hospital USM) having registration number 254.4(1.3) (2012). An oral surgeon conducts all the clinical activity related to the patient treatments from Hospital USM. The flow schematic of the research is shown in Figure 1.

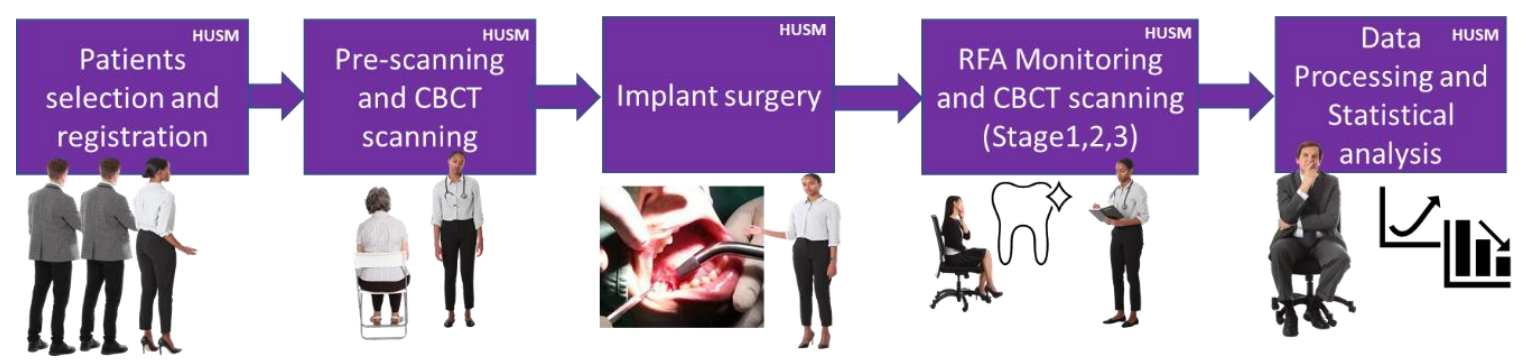

Figure 1 The schematic of research flow and patient treatment

\subsection{Resonance Frequency Analysis (RFA) Method}

Resonance frequency analysis is used to measure implant stability more reliably, and can be conducted at any stage (Konstantinović et al., 2015). Meredith et al. (1997) led the implant measurement using RFA method by developing a device called as Ostell® (1997). The concept of RFA measurement is by measuring the response of frequency resonance and associating it with the micro-movement of the dental implant. The frequency of ultrasound will be transmitted and received through a frequency analyzer to see the frequencies shifting (Hériveaux et al., 2020). The peak of amplitude defines the excitation, which shows a resonance frequency.

In RFA measurement, the measured resonance frequencies are converted into a scale of Implant Stability Quotient (ISQ) with the scale ranging from 0 to 100. Measurement of RFA is affected by the transducer design, contact between the implant and the surrounding bone, and the distance from the marginal bone level. For best measurement, the Smart Peg ${ }^{\mathrm{TM}}$ (the tools together with the Osstell devices which used to measure the resonance frequency) needs to be located at $3 \mathrm{~mm}$ above the soft tissue and perpendicular to the object (Sennerby and Meredith, 2008).

The advantages of RFA method are the consistency of the measurement and that it can be conducted any time at any stage, including the measurement of both primary and secondary implant stability (Juboori et al., 2018). The RFA results can also be associated with other methods such as insertion of torque, bone density, and cortical thickness (Wada et al., 2015).

In this research, the implant stability was measured using RFA device (Osstell, Integration Diagnostic $A B$, Göteborg, Sweden) with the Smart Peg abutment (Integration Diagnostic $\mathrm{AB}$ ). Measurement of the implant stability using this instrument follows the protocols of Barewal et al. (2003) and Bischof et al. (2004). In this procedure, the Smart Pegs were mounted on the implants and tightened by hand with a screw. The transducer was directly connected perpendicularly to the implant, as recommended by the manufacturer, and measured twice from the buccal and lingual side (Guler et al., 2013). The illustration of implant measurement using RFA instrument is shown in Figure 2. 


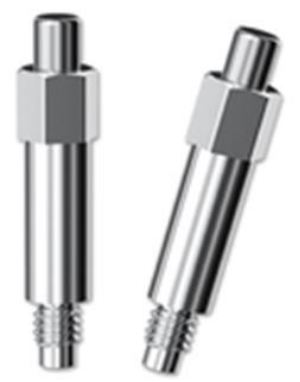

(a)

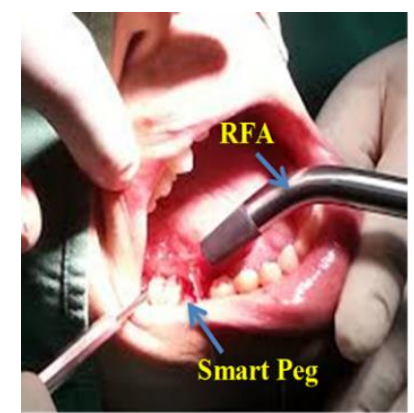

(b)

Figure 2 (a) Implant with a Smart Peg to measure implant stability using RFA; and (b) Measurement of RFA from the buccal and lingual side

In this experiment, the implant stability measured was evaluated based on the schedule shown in Table 1.

Table 1 Evaluation of measurement schedule during implant placement

\begin{tabular}{|c|c|c|c|c|}
\hline Scanning type/Stage: & Pre-Scanning & Stage 1 & Stage 2 & Stage 3 \\
\hline $\begin{array}{l}\text { CBCT scanning } \\
\text { RFA scanning }\end{array}$ & $\begin{array}{l}\text { CBCT scanning } \\
\text { without RFA } \\
\text { (1 week before } \\
\text { implant) }\end{array}$ & $\begin{array}{l}\text { CBCT \& RFA } \\
\text { scanning } \\
\text { (immediate } \\
\text { after implant) }\end{array}$ & $\begin{array}{c}\text { CBCT \& RFA } \\
\text { scanning } \\
\text { (3 months after } \\
\text { implant) }\end{array}$ & $\begin{array}{c}\text { CBCT \& RFA } \\
\text { scanning } \\
\text { (4 months after } \\
\text { implant or } 1 \\
\text { month post- } \\
\text { crown) }\end{array}$ \\
\hline
\end{tabular}

\subsection{Density Measurement}

Density measurements of bone were conducted on CBCT data using MIMICS software, which is available in the Universiti Sains Malaysia. The measurements are pointed at the position of point measurements, as adjusted from the Cemento Enamel Junction (CEJ) position. There are three-point locations of measurements: $6 \mathrm{~mm}, 8 \mathrm{~mm}$, and $12 \mathrm{~mm}$, measured from CEJ level. The example of density measurement at a different stage during the monitoring period is shown in Figure 3.

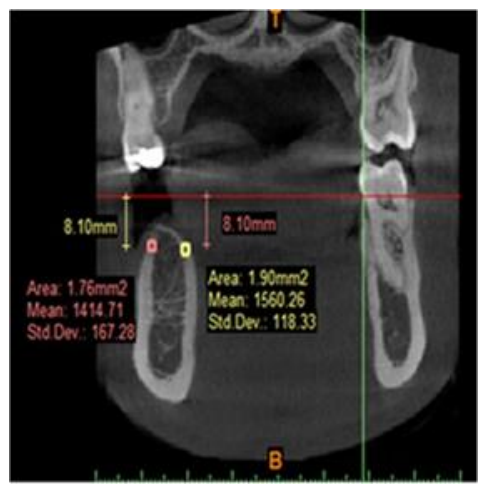

(a)

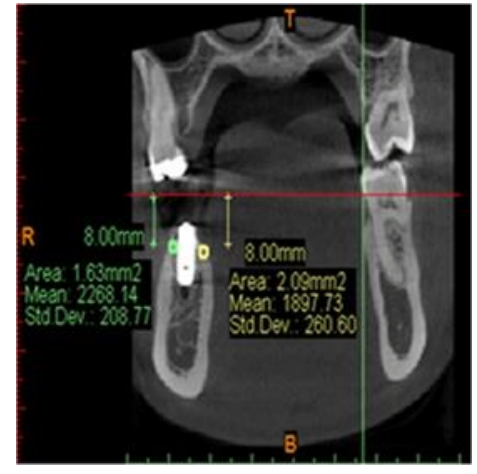

(b)

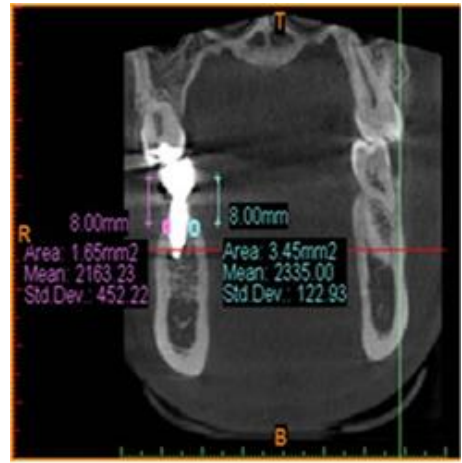

(c)

Figure 3 Density measurement on different stages: (a) Pre-implant; (b) After implant insertion; and (c) After crown insertion. 


\subsection{Population and Samples}

All subjects for dental implant placement undergoing CBCT were obtained from the Hospital USM. Some inclusion criteria have been applied to select patients/participants. The applied criteria are: (i) age range between $25-55$ years; (ii) willing to do the program voluntarily; (iii) single natural tooth edentulous on mandible case; and (iv) the patient who has been edentulous at molar teeth. Meanwhile, the exclusion criteria are a smoker and a person who has a systemic disease such as heart problems, high blood pressure, and diabetes.

The numbers of sample size, which are the minimum number of participants involved in this research, are determined using Stats Direct statistical software V 2.7.8. On the basis of the calculation from the previous researcher, the sample size is derived from the Pearson correlation between CBCT and RFA method (Farre-Pages et al., 2011). The inputs for this calculation are: Power $=90 \%, \alpha=5 \%, \rho=0$, Alt RH $=0.56$. Hence the estimated sample size is 10 patients.

The Statistical Package of Social Science (SPSS) version 18.0 is used for data entry and statistical analysis. A paired $t$-test has been used to compare the measurements of pre-and post-implant groups using either CBCT or RFA.

\section{Results and Discussion}

Implant stability measurements of 10 patients followed the Barewal and Bischof protocol (López et al., 2008). For each patient, implant stabilities have been measured twice in each stage. During measurement, the L-shaped transducer is connected perpendicularly to the implant recommended by the manufacturer to achieve maximum measurement accuracy. Measurements are conducted right after implant placement, pre-crown, and 1 month after the crown is installed. Figure 4 shows the implant placement during surgery and usage of the RFA method to measure the stability of the implant right after its placement. The transducer is directed from the buccal and lingual side to ensure the measurements are capturing implant stability from both the sides. It is assumed that the measured frequency captured in the device is coming from only one side of the implant. Hence the ISQ value for each measurement is merely representing the implant stability occurring on respective sides. Once the total implant stability is obtained, the average of the measurement result from both sides is considered.

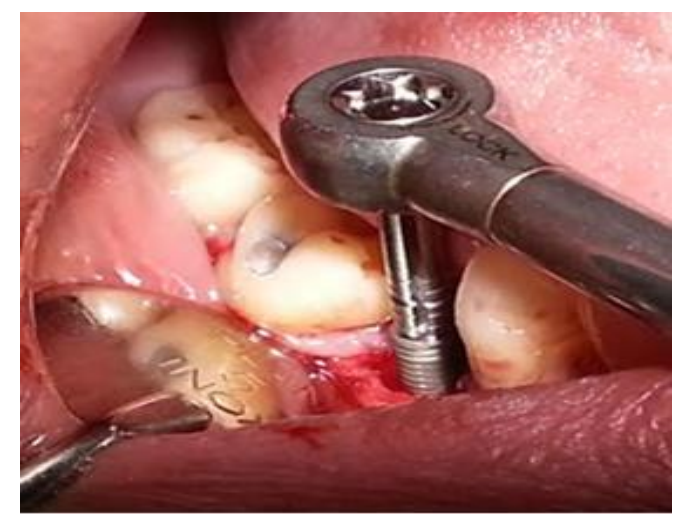

(a)

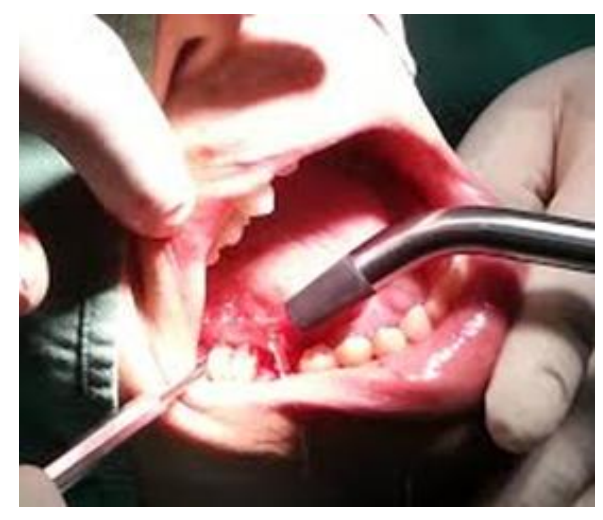

(b)

Figure 4 (a) Implant placement; and (b) Implant stability measurement using RFA Ostell mentor device 
Figure 5 shows the graph of implant stability measurement from 10 patients on 3 different stages (Series 1 = Stage 1, Series 2 = Stage 2, and Series 3 = Stage 3). There are three patients with primary implant stability below 60 ISQ, while the other seven patients have primary implant stability more than 60 ISQ. Implant stability for most of the patients increased, except for one patient showing a decrease. Increasing implant stability is associated with the progress of osseointegration. In this case, it is expected that the implant treatment was successful in nine patients, and only one patient showed problem with implant stability. On the basis of RFA measurement, there are three groups of implant stability progress: increasing implant stability, most constant, and decreasing with the time.

The statistical analysis has been conducted to see the significant difference in measured implant stability between the stages. The study was performed using SPSS by setting the confidence level to $95 \%$. The results show some increment in implant stability from Stage 1 to Stage 2 and Stage 2 to Stage 3. The average implant stability increases from 68.85 ISQ to 77.80 ISQ in Stages 1 and 2, with the different significance $p=0.033$. From Stage 2 to Stage 3, the average implant stability increased from 77.80 ISQ to 82.17 ISQ, with a different significance $p=0.016$.

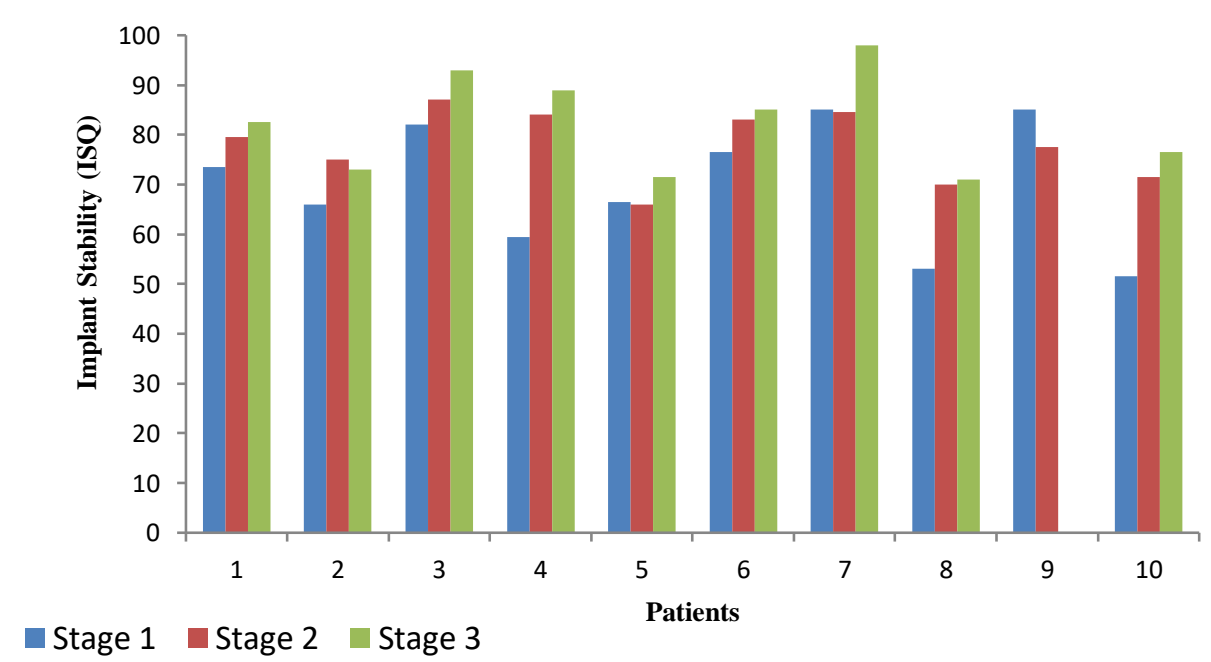

Figure 5 Implant stability of 10 patients on different stages: Stages 1, 2, and 3

The primary implant stability and density of bone are the main factors for the success of implant integration. Hence, during monitoring, the density of the bone around the implant site is also measured. Quality and quantity of bone are estimated based on CBCT data taken in each of the measurement stages. The parameters measured are cortical thickness, bone width, bone height, and density. These parameters are then correlated with measured implant stability to see the relevance and to know the main factor that affects implant stability (Seo et al., 2011). As there are two types of implant stabilities that are measured during monitoring, the correlation is conducted differently. Density in the initial condition (Stage 1), cortical thickness, bone width, and height are correlated with primary implant stability. Meanwhile, the secondary implant stability is related only with respective densities that are measured in each stage (Stages 1 and 2). It was assumed that the primary implant stability is associated with the mechanical engagement of an implant with the surrounding bone resulting in the implant placement.

In contrast, secondary implant stability is considered related to bone regeneration and remodeling during the healing period after implant placement. The mechanism of the jawbone in response to the implant insertion, which is measured as primary and secondary 
implant stability, is not simple. Many factors can affect primary and secondary implant stability, such as the condition of surrounding tissues, bone quantity and quality, implant geometry, and surgical technique. The secondary implant stability is affected by primary implant stability itself, bone remodeling, and implant surface (Javed et al., 2013).

Bone density obtained from CBCT data, which is taken in each stage of measurement, was correlated to implant stability using Pearson's correlation one-tailed test in SPSS software. Figure 6 shows the relationship between the density estimated from CBCT and primary implant stability. The trends of implant stability are similar to that of density; however, the statistical analysis showed that the relationship between both variables has low Pearson's correlation coefficient $(r=0.031)$ with significant $p=0.466$. This statistical analysis indicates no significant correlation between estimated densities from CBCT and primary implant stability obtained from RFA measurement.

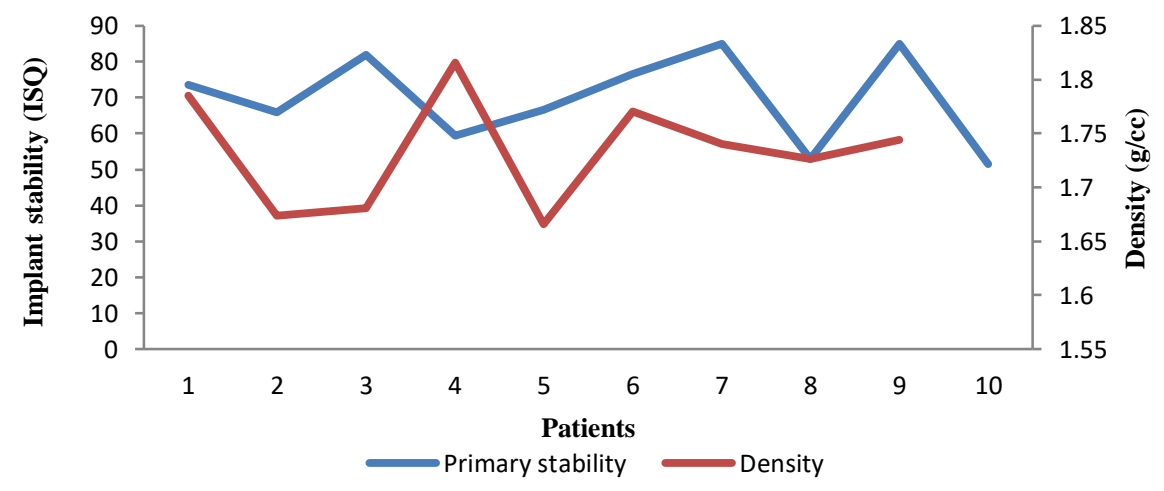

Figure 6 A density and primary implant stability plot that are measured in Stage 1

Secondary implant stability was measured in Stages 2 and 3. These measurements and estimated bone density on each stage are shown together, relatively, in Figure 7. As in the primary implant stability, the secondary implant stability also has a similar trend with the bone density on each stage of measurement. Patients who have high secondary implant stability also have increased bone density. However, statistical analysis shows that the relationship between density and secondary implant stability at Stages 2 and 3 is not significant statistically (Stage 1: Pearson's Correlation $=0.205, p=0.285$; and Stage 2: Pearson's correlation $=-0,018, p=0.482$ ).

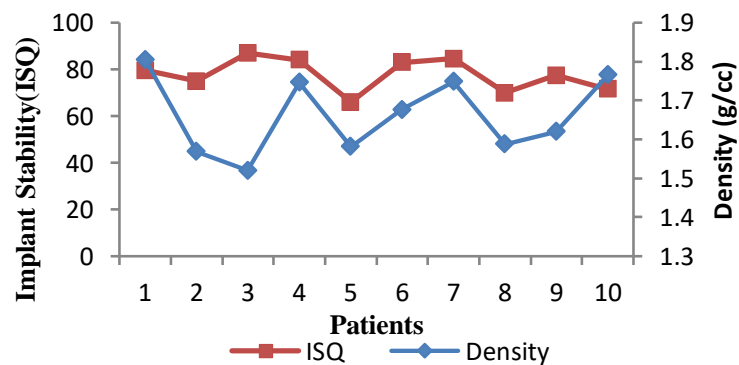

(a)

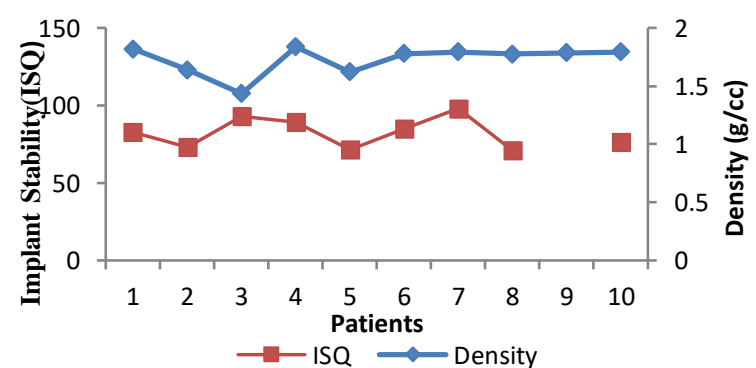

(b)

Figure 7 Progress of implant stability and density changes during the monitoring period: (a) Stage 2; and (b) Stage 3

On the basis of statistical analysis, in the 10 patients involved, there is no significant correlation between bone density and implant stability, either primary or secondary. It is 
clear from the evidence the density was decreasing, but the implant stability on these stages was increasing. In terms of individual correlations with other jaw parameters, implant stability has a good relationship with cortical thickness and bone height. The patient who has a thicker cortex or higher bone has the potential to have high primary implant stability. The mechanical process due to external loading during daily mastication possibly causes an increase in implant stability. Hence, the density decreasing during the healing process (in between Stages 1 and 2) is not lowering implant stability. The increase in implant stability from Stages 1 to 2, could be because of increasing in density or starting of osseointegration. The decreasing and increasing of density during the healing process can be detected by CBCT scanning (Sahib and Al-Adili, 2019).

Primary implant stability was also correlated to the parameters of available space of the jaw, bone height, cortical thickness, and angle insertion. The statistical correlation between primary implant stability with those variables is shown in Table 2 .

Table 2 Pearson Correlation test between implant stability with other variables

\begin{tabular}{lcc}
\hline \multicolumn{1}{c}{ Variable } & Pearson correlation coefficient & $\begin{array}{c}\text { Significance } \\
\text { (one-tailed) }\end{array}$ \\
\hline Bone width & -0.159 & 0.331 \\
Bone height & 0.448 & 0.097 \\
Cortical thickness & 0.272 & 0.224 \\
Angle insertion & -0.173 & 0.355 \\
\hline
\end{tabular}

On the basis of the measurement of all the parameters during the healing period on three different stages, in term of implant stability progress, the patients can be classified under three categories: patients who have a significant increment in their implant stability during the healing process; the patient who has a constant implant stability progress or the growth is not substantial; and the patient who has negative implant stability progress, where the implant stability of the patient decreases during the healing process.

It is interesting that in future, the parameters affecting implant stability can be evaluated together with other dynamic properties such as stress, strain, and displacement of the implant during dynamic process and loading, such as daily mastication. Hence, the implant stability is not only correlated with the static parameters, but also can be connected with those dynamic properties for better understanding and monitoring of the implant biomechanically (Iridiastadi et al., 2020).

\section{Conclusions}

The monitoring of a dental implant system of a patient after surgery is needed to evaluate the progress of osseointegration. On the basis of the monitoring of implant stability on three different stages, the success of implant treatment is achieved. The increment of implant stability indicated those achievement of implant treatment. In general, implant stability increased from Stages 1 to 2 with an increment from 68.85 to 77.80 ISQ, and from Stage 2 to 3 with an increment from 77.80 to 82.17 in ISQ scale. The implant stability, either the primary or secondary, is not statistically correlated to bone density. However, the available space for the implant site, that is the bone height of mandible and cortical thickness, is associated strongly with the implant stability of the patient. The patient who has a thicker cortex or higher bone can have high primary implant stability.

Density of bone during healing stage will change due to the modeling and remodeling processes; however the measurement of density to indicate implant stability 
is still challenging. To improve the correlation between density and implant stability measurements, in future work, more patients with different races should be involved. Besides that, the implant stability can also be correlated with other dynamic parameters such as stress distribution and displacement of site implant to better understand the integrated biomechanical evaluation of jaw system.

\section{References}

Barewal, R.M., Oates, T.W., Meredith, N., Cochran, D.L., 2003. Resonance Frequency Measurement of Implant Stability In Vivo on Implants with a Sandblasted and AcidEtched Surface. The International Journal of Oral \& Maxillofacial Implants, Volume 18(5), pp. 641-651

Bischof, M., Nedir, R., Szmukler-Moncler, S., Bernard, J.-P., Samson, J., 2004. Implant Stability Measurement of Delayed and Immediately Loaded Implants during Healing. Clinical Oral Implants Research, Volume 15(5), pp. 529-539

Chang, P.-C., Lang, N.P., Giannobile, W.V., 2010. Evaluation of Functional Dynamics during Osseointegration and Regeneration Associated with Oral Implants: A Review. Clinical Oral Implants Research., Volume 21(1), pp. 1-12

Farre-Pages, N., Auge-Castro, M., Alaejos-algarra, F., Mareque-Bueno, J., Ferres-Padro, E., Hernandez-Alfaro, F., 2011. Relation between Bone Density and Primary Implant Stability. Medicina Oral Patología Oral y Cirugia Bucal, Volume 16(1), pp. e62-e67

Guler, A.U., Sumer, M., Duran, I., Sandikci, E.O., Telcioglu, N.T., 2013. Resonance Frequency Analysis of 208 Straumann Dental Implants during the Healing Period. The Journal of Oral Implantology, Volume 39(2), pp. 161-167

Hériveaux, Y., Audoin, B., Biateau, C., Nguyen, V.H., Haïat, G., 2020. Ultrasonic Propagation in a Dental Implant. Ultrasound in Medicine and Biology, Volume 46(6), pp. 1464-1473

Iridiastadi, H., Vani, T., Yamin, P.A.R., 2020. Biomechanical Evaluation of a Patient-Handling Technology Prototype. International Journal of Technology, Volume 11(1), pp. 180-189

Javed, F., Ahmed, H.B., Crespi, R., Romanos, G.E., 2013. Role of Primary Stability for Successful Osseointegration of Dentqualityal Implants: Factors of Influence and Evaluation. Interventional Medicine \& Applied Science, Volume 5, pp. 162-167

Juboori, M.J.AL, Attas, M.A.AL, Gomes, R.Z., Alanbari, B.F., 2018. Using Resonance Frequency Analysis to Compare Delayed and Immediate Progressive Loading for Implants Placed in the Posterior Maxilla: A Pilot Study. The Open Dentistry Journal, Volume 12(1), pp. 801-810

Konstantinović, V.S., Ivanjac, F., Lazić, V., Djordjević, I., 2015. Assessment of Implant Stability by Resonant Frequency Analysis. Vojnosanitetski Pregled, Volume 72(2), pp. 169-174

López, A.B., Martínez, J.B., Pelayo, J.L., García, C.C., Peñarrocha, M., 2008. Resonance Frequency Analysis of Dental Implant Stability during the Healing Period. Medicina Oral, Volume 13(4), pp. 2-5

Meredith, N., Science, D., Maudlin, L., 1997. Resonance Frequency Measurements of Implant Stability in Viva. A Cross-Sectional and Longitudinal Study of Resonance Frequency Measurements on Implants in the Edentulous and Partially Dentate Maxilla., Clinical Oral Implants Research, Volume 8(3), pp. 226-233

Rabel, A., Kohler, S.G., Westhausen, A.M.S., 2007. Clinical Study on the Primary Stability of Two Dental Implant Systems with Resonance Frequency Analysis. Clinical Oral Investigations, Volume 11(3), pp. 257-265

Sahib, A.M., Al-Adili, S.S., 2019. Evaluation of Healing Process of Periapical Defect Filled by Platelet Rich Fibrin using Cone Beam Computed Tomography-Comparative Clinical 
Study. Indian Journal of Public Health Research and Development, Volume 10(6), pp. 448-453

Sennerby, L., Meredith, N., 2008. Implant Stability Measurements using Resonance Frequency Analysis : Biological and Biomechanical Aspects and Clinical Implications. Periodontology 2000, Volume 47(1), pp. 51-66

Seo, T., Song, B., Seo, K., Cho, J., Yoon, G., 2011. A Study of Optimization of Machining Conditions in Micro End-Milling by using Response Surface Design. International Journal of Technology, Volume 2(3), pp. 248-256

Staden, V., Guan, H., Loo, Y.C., 2006. Application of Finite Element Method in Dental Implant Research. Computer Methods in Biomechanics and Biomedical Engineering, Volume 9(4), pp. 257-270

Supriadi, S., Sitanggang, T.W., Irawan S,B., Suharno, B., Kiswanto, G., Prasetyadi, T., 2015. Orthodontic Bracket Fabrication using the Investment Casting Process. International Journal of Technology, Volume 6(4), pp. 613-621

Swami, V., Vijayaraghavan, V., Swami, V., 2016. Current Trends to Measure Implant Stability. Journal of Indian Prosthodontic Society, Volume 16(2), pp. 124-130

Turkyilmaz, I., Mcglumphy, E.A., 2008. Influence of Bone Density on Implant Stability Parameters and Implant Success: A Retrospective Clinical Study. BMC Oral Health, Volume 8(32), pp. 1-9

Wada, M., Tsuiki, Y., Suganami, T., Ikebe, K., Sogo, M., Okuno, I., 2015. The Relationship between the Bone Characters Obtained by CBCT and Primary Stability of the Implants. International Journal of Implant Dentistry, Volume 1(2), pp. 1-7 